\title{
Inhibidores directos del Factor Xa para la prevención del tromboembolismo en pacientes sometidos a reemplazo total de cadera o de rodilla. Protocolo de una revisión sistemática
}

\author{
Ignacio Neumann ${ }^{1,2,3^{*}}$, Gabriel Rada ${ }^{2,3}$, Juan Carlos Claro ${ }^{2,3}$, Alonso Carrasco-Labra ${ }^{1,4}$, Kristian Thorlund , \\ Elie A. Akl ${ }^{1,5,6}$, Shannon M. Bates ${ }^{7,8}$, Gordon H. Guyatt ${ }^{1,8}$
}

\begin{abstract}
Resumen - Los reemplazos articulares de cadera y la rodilla se encuentran entre los procedimientos quirúrgicos más comunes en América del Norte y Europa y están aumentando en frecuencia. La enfermedad tromboembólica es la complicación médica más frecuente en este tipo de pacientes. Por esta razón, las guías de práctica clínicas actuales recomiendan la tromboprofilaxis de rutina con heparinas de bajo peso molecular (HBPM), antagonistas de la vitamina K (AVK) o pentasacáridos sintéticos (fondaparinux) después de estos procedimientos.
\end{abstract}

Palabras claves: cadera; reemplazo; quirúrgico; rodilla; tromboembólica.

Abstract - Hip and Knee replacements are among the most common surgical procedures in North America and Europe, and are increasing its occurrency. Tromboembolic disease is the most frequent medical complication in patients submitted to these procedures. Due to this reason, clinical practice guidelines suggest rutinary tromboprofilaxis with low molecular weight heparines (LMWH), Vitamin K antagonists (VKA) or synthetic pentasaccharides (fondaparinux) after these procedures.

Keywords: Hip, replacement, surgical, knee, tromboembolic.

Fecha de envío: 15 de Mayo de 2012 - Fecha de aceptación: 20 de Septiembre de 2012

\section{Introducción}

Los reemplazos articulares de cadera y la rodilla se encuentran entre los procedimientos quirúrgicos más comunes en América del Norte y Europa (OECD; NCHS, 2010) y están aumentando en frecuencia (Dixon et al., 2004; Kurtz et al., 2005; CIFHI, 2008). La enfermedad tromboembólica es la complicación médica más frecuente en este tipo de pacientes (Phillips et al., 2003; Zhan et al., 2007). Por esta razón, las guías de práctica clínicas actuales recomiendan la tromboprofilaxis de rutina con heparinas de bajo peso molecular (HBPM), antagonistas de la vitamina K (AVK) o pentasacáridos sintéticos (fondaparinux) después de estos procedimientos (Geerts et al., 2008; Johanson et al., 2009).
La profilaxis con HBPM o fondaparinux es muy eficaz en reducir la incidencia de tromboembolismo venoso después de un reemplazo de cadera o rodilla (Geerts et al., 2008). Sin embargo, el paciente debe tolerar inyecciones subcutáneas diarias. Los antagonistas de la vitamina K, por otra parte, son la única opción disponible por la vía oral, pero resultan menos eficaces que las HBPM (Mismetti et al., 2004) y requieren de un control cuidadoso (Hirsh, 1991).

Las limitaciones de los anticoagulantes actuales han dado lugar al desarrollo de nuevos fármacos (Bates \& Weitz, 2006), incluyendo los inhibidores orales directos del Factor $X$ activado (inhibidores FXa). Esta familia de nuevas drogas tiene las

1) Departamento de Epidemiología Clínica y Bioestadística. Universidad de McMaster, Hamilton, Canadá. 2) Programa de Salud Basado en Evidencias, Facultad de Medicina, Pontificia Universidad Católica de Chile, Santiago, Chile. 3) Hospital Dr. Sótero del Río, Puente Alto, Chile. 4) Unidad de Odontología Basada en Evidencias, Facultad de Odontología, Universidad de Chile, Chile. 5) Departamento de Medicina, Universidad Estatal de Nueva York, Búfalo, NY, USA. 6) Departamento de Medicina Familiar, Universidad Estatal de Nueva York, Búfalo, NY, USA. 7) Instituto de Investigación en Trombosis y Arterioesclerosis, Hamilton, Ontario, Canadá. 8) Departamento de Medicina, Universidad de McMaster, Hamilton, Canadá.

*Autor de correspondencia: ignacio.neumann@gmail.com 
ventajas de la administración oral y de una farmacocinética y respuesta anticoagulante predecible (Mueck et al., 2008).

Tres revisiones sistemáticas han evaluado el efecto de los inhibidores FXa en pacientes sometidos a reemplazo total de cadera o rodilla. Dos de estas revisiones (Cao et al., 2010; Turun et al., 2011) incluyeron los mismos ocho estudios que compararon rivaroxaban versus enoxaparina en pacientes sometidos a reemplazo de cadera o rodilla. Los autores de estas revisiones concluyeron que rivaroxaban es superior a enoxaparina basándose en el efecto sobre un outcome compuesto de tromboembolismo venoso sintomático y asintomático. La tercera revisión (Huang et al., 2011) incluyó tres estudios que evaluaron apixaban versus enoxaparina en pacientes sometidos a reemplazo total de rodilla. Los autores no encontraron ningún efecto de apixaban en mortalidad, pero sí una reducción en el riesgo de un outcome compuesto de trombosis venosa profunda (TVP) sintomática y asintomática.

Las revisiones sistemáticas existentes tienen múltiples limitaciones. En ellas solo se exploran comparaciones específicas de dos drogas, se pone el foco en eventos asintomáticos detectados a través de venografía rutinaria, sin reportar los efectos absolutos sobre outcomes importantes para los pacientes (como tromboembolismo sintomático) y no se exploran los efectos de las diferentes dosis. Por estas razones, se decidió realizar una nueva revisión sistemática con un enfoque más amplio (Gotzsche, 2000), que proporcionara una estimación de los efectos absolutos sobre los outcomes importantes para los pacientes (Guyatt et al., 2011) y que permitiera explorar los efectos de las distintas dosis.

\section{Métodos}

\section{Criterios de inclusión y exclusión:}

Se utilizarán los siguientes criterios de inclusión:

- Diseño del estudio: cualquier ensayo clínico randomizado. - Población: individuos sometidos a reemplazo de cadera o rodilla por cualquier causa.

- Intervención:Inhibidores FXa, incluyendo: rivaroxaban, apixaban, edoxabán, YM150, TAK442, betrixaban y LY517717.

- Comparación: cualquier tromboprofilaxis farmacológica o no farmacológica.

- Outcomes: reporte de al menos uno de los siguientes outcomes: mortalidad al final de la profilaxis, mortalidad durante el período de seguimiento, TVP sintomática, Tromboembolismo Pulmonar (TEP), hemorragia mayor (según lo definido por los autores de cada ensayo), hemorragia intracraneal y sangrado que conduce a la reintervención.

Se excluirán del análisis los estudios que

- Reporten los outcomes de la revisión como compuestos sin dar información de los componentes.

- Presenten comparaciones en que dos variables están siendo evaluadas a la vez (por ejemplo, diferentes drogas con diferentes tiempos de exposición).

\section{Búsqueda}

Realizaremos búsquedas electrónicas, sin restricciones de idioma, en las siguientes bases de datos a partir de su fecha de inicio: MEDLINE, EMBASE y el Registro Cochrane Central de Ensayos Controlados (CENTRAL).

La estrategia de búsqueda se desarrolló con la ayuda de una bibliotecaria con experiencia en búsquedas de revisiones sistemáticas, utilizando la combinación de términos que describan la intervención y la población de interés con un filtro para estudios clínicos randomizados (en el Apéndice 1 se presenta la estrategia de búsqueda completa).

Adicionalmente, para identificar estudios potencialmente pasados por alto en la búsqueda electrónica, dos revisores (AC, IN) de forma independiente revisarán todas las referencias de los estudios incluidos y de las revisiones (narrativas y sistemáticas) identificadas mediante la búsqueda electrónica.

Por último, con el fin de identificar estudios no publicados, dos revisores (JCC, IN) realizarán de forma independiente una búsqueda manual en los resúmenes de las siguientes conferencias: "Congress of the International Society on Thrombosis and Hemostasis" (del 2003 al 2010) y "Annual Proceedings of the American Society of Hematology" (del 2004 al 2010).

\section{Selección de estudios:}

A partir de las referencias identificadas por la búsqueda, dos revisores $(G R, I N)$ realizarán de forma independiente la selección de estudios utilizando los criterios de inclusión y exclusión. Cualquier referencia identificada por alguno de estos revisores como potencialmente relevante será evaluada a texto completo. Luego, mediante un formulario estandarizado y piloteado, los mismos revisores identificaran los artículos elegibles para esta revisión. El acuerdo entre los 2 revisores para la etapa de selección de texto completo se evaluará mediante la estadística kappa no ponderada (к). 


\section{Extracción de datos:}

Dos revisores $(G R, I N)$ en forma independiente realizarán la extracción de datos. Antes de proceder con el análisis de la información, los datos extraídos por ambos revisores serán comparados y se resolverán los desacuerdos en consenso.

Se recogerá la siguiente información de los estudios incluidos:

- Características basales de los pacientes incluidos: edad promedio, proporción de mujeres en la población de estudio, índice de masa corporal promedio, tipo de cirugía, duración de la cirugía, duración de la estadía hospitalaria, proporción de la población con antecedentes de tromboembolismo.

- Características de la intervención y control: droga utilizada, dosis, duración, vía deadministracióny tiempo de la primera dosis.

- Outcomes: número de eventos y número de pacientes analizados para cada uno de los outcomes de esta revisión.

\section{Evaluación del riesgo de sesgo de los estudios incluidos:}

Dos revisores $(G R, I N)$ en forma independiente evaluarán el riesgo de sesgo de los estudios incluidos. Con este objetivo, se seguirán los procedimientos sugeridos por la herramienta "Risk of Bias" de la colaboración Cochrane (Bradburn et al., 2007; Friedrich et al., 2007). Para los efectos de esta revisión, estudios con "bajo riesgo de sesgo" se definen como los estudios sin sesgo en todos los dominios de la herramienta "Risk of Bias"; estudios con "riesgo de sesgo incierto" se definen como los estudios con riesgo de sesgo incierto para uno o más dominios; y estudios con "alto riesgo de sesgo" como la presencia de sesgo potencial de un o más dominios. Se evaluará la concordancia entre los 2 revisores para la evaluación de riesgo de sesgo con la estadística kappa ponderada ( $\mathrm{k}$ ).

En caso de ser necesario, se intentará establecer contacto con los autores para obtener información adicional.

\section{Análisis}

Las tres revisiones sistemáticas que han evaluado los efectos de los inhibidores FXa en la tromboprofilaxis de pacientes sometidos a reemplazo de cadera o rodilla sugieren que el riesgo de los outcomes importantes para los pacientes es muy bajo (menos del 1\%). Nosotros esperamos un escenario similar en esta revisión.

Estudios de simulación han demostrado que en presencia de eventos de muy baja frecuencia (menos del 1\%) los métodos más frecuentes de meta-análisis (el método de de DerSimonian y Laird, y el de Mantel-Haenszel) pueden producir sesgo en las estimaciones del efecto y los intervalos de confianza correspondientes. El método de Peto odds ratio parece ser el método menos sesgado y el más eficaz para meta analizar eventos infrecuentes (Bradburn et al., 2007).

Por otra parte, también anticipamos estudios sin eventos en ambos grupos ("estudios de cero eventos"). Tradicionalmente, este tipo de estudios se excluyen de los meta-análisis dado que no aportan información sobre la dirección y magnitud relativa del efecto de los tratamiento. Sin embargo, algunos autores argumentan a favor de la inclusión de este tipo de estudios utilizando las llamadas correcciones de continuidad, ya que con este método se obtiene una estimación más conservadora del efecto, se aumenta la precisión y se puede disminuir la heterogeneidad (Friedrich et al., 2007).

Aunque ningún método ofrece una estimación totalmente libre de sesgo cuando los eventos son raros, realizaremos el análisis primario de esta revisión utilizando el método de Peto excluyendo los estudios con cero eventos, y realizaremos análisis de sensibilidad con otros métodos de meta análisis incluyendo los estudios de cero eventos con diferentes correcciones de continuidad (ver análisis de sensibilidad).

Utilizaremos el software RevMan 5.1 para realizar los metaanálisis y el software GRADEpro 3.6 para resumir los resultados en una tabla de evidencia (llamada perfil de evidencias) y estimar la confianza en los estimadores del efecto (Guyatt et al., 2008). Además de analizar el efecto relativo de los inhibidores FXa en comparación con otras alternativas, vamos a estimar su efecto absoluto directamente meta-analizando las reducciones de riesgo absoluto observadas en cada estudio. El resultado de este análisis será expresado en frecuencias naturales para facilitar su comprensión (Akl et al., 2011).

En el caso de la TVP sintomática, el uso de venografía de rutina en los estudios randomizados puede sesgar la estimación del riesgo absoluto del evento, ya que por un lado la venografía detecta pacientes potencialmente sintomáticos en etapas tempranas que no alcanzan a desarrollar síntomas (se subestima el riesgo del evento sintomático), y por otro, en presencia de una venografía positiva, síntomas menores que normalmente serían ignorados pueden llevar a catalogar el evento como "sintomático" (a su vez, se sobreestima el riesgo del evento sintomático). Por esta razón, además vamos a utilizar información de estudios observacionales para estimar el efecto absoluto de los inhibidores FXa en el outcome TVP sintomática. 


\section{Heterogeneidad y análisis de subgrupos}

Vamos a evaluar la heterogeneidad mediante el test Chi-cuadrado y la estadística $\mathrm{I}^{2}$.

Independientemente del resultado de los test de heterogeneidad, los siguientes subgrupos serán analizados:

1. Riesgo de sesgo: evaluado por la herramienta "Risk of Bias" de la colaboración Cochrane. Vamos a comparar las categorías: bajo riesgo de sesgo vs. riesgo de sesgo incierto vs. alto riesgo de sesgo. Estimamos que un bajo riesgo de sesgo se asocia con un tamaño del efecto más pequeño.

2. Droga utilizada en el grupo intervención: exploraremos las siguientes categorías: rivaroxaban vs. apixaban vs. otros. Estimamos que no existirán diferencias entre las drogas.

3. Dosis de la intervención: clasificaremos la dosis de los inhibidores FXa en alta, intermedia y baja de acuerdo con el rango utilizado en los estudios incluidos. Nuestra hipótesis es que las dosis más altas serían más eficaces en la prevención de eventos trombóticos, pero aumentarían el riego de hemorragia.

4. Duración de la profilaxis: a corto plazo (14 días o menos) vs. profilaxis extendida (más de 14 días). Planteamos la hipótesis de que la profilaxis extendida sería más eficaz en la prevención de de eventos trombóticos, pero puede aumentar el riesgo de hemorragia.

En caso de encontrar más de un subgrupo estadísticamente significativo con el test Chi-cuadrado, se realizará una metaregresión para comprobar la independencia del efecto de subgrupo.

Este análisis será llevado a cabo en con el software SAS 9.2.

\section{Evaluacion del sesgo de publicación}

Evaluaremos la probabilidad de sesgo de publicación gráficamente mediante el uso de Funnel plots.

\section{Manejo de la Información faltante}

Intentaremos contactar a los autores de los estudios incluidos para obtener información adicional a la publicada toda vez que esto parezca necesario. El meta-análisis principal se realizará considerando toda la información disponible en la publicaciones de los estudios incluidos así como la obtenida mediante el contacto con los autores. En caso de existir información faltante respecto de los outcomes de esta revisión, se llevará a cabo un análisis de sensibilidad con supuestos extremos respecto de la información faltante (ver a continuación).

\section{Análisis de sensibilidad}

1. Impacto de la información faltante: para estimar el impacto de la información faltante, se realizará un análisis con supuestos extremos respecto de la información faltante en el grupo intervención (inhibidores FXa):

- Los participantes no reportados en el grupo intervención tienen dos veces la probabilidad de presentar el outcome que los participantes reportados. - Los participantes no reportados en el grupo intervención tienen tres veces la probabilidad de presentar el outcome que los participantes reportados.

En ambos casos, se asumirá que los pacientes no reportados en el grupo control tienen la misma probabilidad de presentar el outcome que los reportados.

2. Método de análisis: repetiremos el meta-análisis utilizando el método de Mantel-Haenszel con diferentes correcciones de continuidad: 0,5 constante para ambos grupos, el inverso del tamaño del grupo y la corrección de continuidad empírica propuesta por Sweeting et al. (2004).

\section{Referencias}

Akl EA, Oxman AD, Herrin J, Vist GE, Terrenato I, Sperati F, Costiniuk C, Blank D \& Schunemann H. (2011). Using alternative statistical formats for presenting risks and risk reductions. The Cochrane database of systematic reviews, CD006776.

Bates SM \& Weitz Jl. (2006). The status of new anticoagulants. British journal of haematology 134, 3-19.

Bradburn MJ, Deeks JJ, Berlin JA \& Russell Localio A. (2007). Much ado about nothing: a comparison of the performance of metaanalytical methods with rare events. Statistics in medicine 26, 53-77.

Cao YB, Zhang JD, Shen H \& Jiang YY. (2010). Rivaroxaban versus enoxaparin for thromboprophylaxis after total hip or knee arthroplasty: a meta-analysis of randomized controlled trials. European journal of clinical pharmacology 66, 1099-1108.

CIFHI. (2008). Hip and Knee Replacements in Canada 2007, Annual Report., ed. CIFHI. Ottawa.

Dixon T, Shaw M, Ebrahim S \& Dieppe P. (2004). Trends in hip 
and knee joint replacement: socioeconomic inequalities and projections of need. Annals of the rheumatic diseases 63, 825-830.

Friedrich JO, Adhikari NK \& Beyene J. (2007). Inclusion of zero total event trials in meta-analyses maintains analytic consistency and incorporates all available data. BMC medical research methodology 7,5 .

Geerts WH, Bergqvist D, Pineo GF, Heit JA, Samama CM, Lassen MR, Colwell CW \& American College of Chest P. (2008). Prevention of venous thromboembolism: American College of Chest Physicians Evidence-Based Clinical Practice Guidelines (8th Edition). Chest 133, 381S-453S.

Gotzsche PC. (2000). Why we need a broad perspective on metaanalysis. It may be crucially important for patients. Bmj 321, 585-586.

Guyatt GH, Oxman AD, Kunz R, Atkins D, Brozek J, Vist G, Alderson P, Glasziou P, Falck-Ytter Y \& Schunemann HJ. (2011). GRADE guidelines: 2 . Framing the question and deciding on important outcomes. Journal of clinical epidemiology 64, 395-400.

Guyatt GH, Oxman AD, Vist GE, Kunz R, Falck-Ytter Y, AlonsoCoello P, Schunemann HJ \& Group GW. (2008). GRADE: an emerging consensus on rating quality of evidence and strength of recommendations. Bmj 336, 924-926.

Hirsh J. (1991). Oral anticoagulant drugs. The New England journal of medicine 324, 1865-1875.

Huang J, Cao Y, Liao C, Wu L \& Gao F. (2011). Apixaban versus enoxaparin in patients with total knee arthroplasty. A meta-analysis of randomised trials. Thrombosis and haemostasis 105, 245-253.

Johanson NA, Lachiewicz PF, Lieberman JR, Lotke PA, Parvizi J, Pellegrini V, Stringer TA, Tornetta P, 3rd, Haralson RH, 3rd \& Watters WC, 3rd. (2009). Prevention of symptomatic pulmonary embolism in patients undergoing total hip or knee arthroplasty. The Journal of the American Academy of Orthopaedic Surgeons 17, 183-196.

Kurtz S, Mowat F, Ong K, Chan N, Lau E \& Halpern M. (2005). Prevalence of primary and revision total hip and knee arthroplasty in the United States from 1990 through 2002. The Journal of bone and joint surgery American volume 87, 1487-1497.

Mismetti P, Laporte S, Zufferey P, Epinat M, Decousus H \& Cucherat M. (2004). Prevention of venous thromboembolism in orthopedic surgery with vitamin $\mathrm{K}$ antagonists: a meta-analysis. Journal of thrombosis and haemostasis: JTH 2, 1058-1070.

Mueck W, Eriksson BI, Bauer KA, Borris L, Dahl OE, Fisher WD,
Gent M, Haas S, Huisman MV, Kakkar AK, Kalebo P, Kwong LM, Misselwitz F \& Turpie AG. (2008). Population pharmacokinetics and pharmacodynamics of rivaroxaban--an oral, direct factor Xa inhibitor--in patients undergoing major orthopaedic surgery. Clinical pharmacokinetics 47, 203-216.

NCHS. (2010). Health, United States, 2009: With Special Feature on Medical Technology. Hyattsville, MD.

OECD. Health at a Glance: Europe 2010. OECD Publishing.

Phillips CB, Barrett JA, Losina E, Mahomed NN, Lingard EA, Guadagnoli E, Baron JA, Harris WH, Poss R \& Katz JN. (2003). Incidence rates of dislocation, pulmonary embolism, and deep infection during the first six months after elective total hip replacement. The Journal of bone and joint surgery American volume 85-A, 20-26.

Sweeting MJ, Sutton AJ \& Lambert PC. (2004). What to add to nothing? Use and avoidance of continuity corrections in metaanalysis of sparse data. Statistics in medicine 23, 1351-1375.

Turun S, Banghua L, Yuan Y, Zhenhui L, Ying N \& Jin C. (2011). A systematic review of rivaroxaban versus enoxaparin in the prevention of venous thromboembolism after hip or knee replacement. Thrombosis research 127, 525-534.

Zhan C, Kaczmarek R, Loyo-Berrios N, Sangl J \& Bright RA. (2007). Incidence and short-term outcomes of primary and revision hip replacement in the United States. The Journal of bone and joint surgery American volume 89, 526-533.

\section{Apéndice 1 - Estrategias de búsqueda}

\section{A1. MEDLINE:}

1. randomized controlled trial.pt.

2. controlled clinical trial.pt.

3. randomized.ab.

4. placebo.ab.

5. drug therapy.fs.

6. randomly.ab.

7. trial.ab.

8. groups.ab.

9. 1 or 2 or 3 or 4 or 5 or 6 or 7 or 8

10. exp animals/ not humans.sh.

11.9 not 10

12. rivaroxaban.mp.

13. Xarelto.mp.

14. BAY 59-7939.mp. 
15. apixaban.mp.

16. BMS-562247.mp.

17. edoxaban.mp.

18. DU-176b.mp.

19. betrixaban.mp.

20. YM150.mp.

21. TAK-442.mp.

22. LY517717.mp.

23. PD0348292.mp.

24. exp Factor Xa/

25. Factor Xa.mp.

26. 12 or 13 or 14 or 15 or 16 or 17 or 18 or 19 or 20 or 21 or 22

or 23 or 24 or 25

27. exp Arthroplasty/

28. arthroplast*.mp.

29. exp Joint Prosthesis/

30. prosthe $^{*} \cdot \mathrm{mp}$.

31. replac*.mp.

32. knee.mp.

33. hip.mp.

34.27 or 28 or 29 or 30 or 31 or 32 or 33

35.11 and 26 and 34

\section{A2. EMBASE}

1. Clinical trial/

2. Randomized controlled trial/

3. Randomization/

4. Single blind procedure/

5. Double blind procedure/

6. Crossover procedure/

7. Placebo/

8. Randomi?ed controlled trial\$.tw.

9. Rct.tw.

10. Random allocation.tw.

11. Randomly allocated.tw.

12. Allocated randomly.tw.

13. (allocated adj2 random).tw.

14. Single blind\$.tw.

15. Double blind\$.tw.

16. ((treble or triple) adj blind\$).tw.

17. Placebo\$.tw.

18. Prospective study/

19. or/1-18

20. Case study/

21. Case report.tw.

22. Abstract report/ or letter/

23. or/20-22
24. 19 not 23

25. exp RIVAROXABAN/

26. rivaroxaban.mp.

27. Xarelto.mp.

28. BAY 59-7939.mp.

29. exp APIXABAN/

30. apixaban.mp.

31. BMS-562247.mp.

32. edoxaban.mp.

33. DU-176b.mp.

34. $\exp$ BETRIXABAN/

35. betrixaban.mp.

36. YM150.mp.

37. TAK-442.mp.

38. LY517717.mp.

39. PD0348292.mp.

40. exp blood clotting factor $10 \mathrm{a} /$

41. Factor Xa.mp.

42.25 or 26 or 27 or 28 or 29 or 30 or 31 or 32 or 33 or 34 or 35 or 36 or 37 or 38 or 39 or 40 or 41

43. $\exp$ ARTHROPLASTY/

44. arthroplast*.mp.

45. exp joint prosthesis/

46. prosthe*.mp.

47. replac*.mp.

48. knee.mp.

49. hip.mp.

50.43 or 44 or 45 or 46 or 47 or 48 or 49

51.24 and 42 and 50

\section{A3. CENTRAL}

1. rivaroxaban

2. BAY 59-7939

3. apixaban

4. BMS-562247

5. edoxaban

6. DU-176b

7. betrixaban

8. YM150

9. TAK-442

10. LY517717

11. PD0348292

12. Factor Xa

13. 1 or 2 or 3 or 4 or 5 or 6 or 7 or 8 or 9 or 10 or 11 or 12 or 13

14. Arthroplasty

15. Joint Prosthesis

16. knee 
Neuman et al. 2012

17. hip

18. 14 or 15 or 16 or 17

19. 13 and 18 